\title{
OPTIMIZACIÓN Y CONTROL EN CASCADA DE TEMPERATURA DE RECINTO MEDIANTE SISTEMAS DE REFRIGERACIÓN
}

\author{
David Rodríguez, José A. Alfaya, Guillermo Bejarano, Manuel G. Ortega \\ Departamento de Ingeniería de Sistemas y Automática, Universidad de Sevilla \\ $\{$ drgarcia, jalonso9, gbejarano, mortega $\}$ us.es
}

\section{Resumen}

Este artículo analiza la eficiencia energética de un ciclo de refrigeración, generando un optimizador de referencias para el control basado en aproximaciones lineales. Se plantean dos enfoques de control: el control de potencia, en el cual se propone un controlador robusto descentralizado para la regulación de la potencia frigorífica dada por el ciclo, y el control de temperatura de recinto, en el cual se considera también la dinámica del recinto a refrigerar y se propone una estructura de control en cascada donde un controlador externo genera las referencias de potencia frigorífica al controlador interno del ciclo de refrigeración. Se analizan en simulación y también experimentalmente el seguimiento de referencias y el rechazo de perturbaciones en ambas estrategias de control.

Palabras clave: Sistemas de refrigeración, Optimización estática, Control en cascada, Planta experimental.

\section{INTRODUCCIÓN}

El ciclo de refrigeración por compresión de vapor constituye el método más extendido para la generación de frío. Estos sistemas se utilizan en áreas tan diversas como regulación de la temperatura en estancias habitadas, almacenamiento y transporte de alimentos y múltiples procesos industriales. Dado el considerable impacto causado por el consumo energético de estos sistemas en los balances económicos y medioambientales, detallado por múltiples informes $[9,15,16]$, así como teniendo en cuenta la escasez creciente de fuentes de energía fósiles y el desarrollo todavía lento de tecnologías de energía renovable, la operación óptima en términos de eficiencia energética de los sistemas de refrigeración por compresión de vapor existentes se presenta como un problema clave.

En un ciclo de refrigeración se extrae energía térmica del recinto a refrigerar (en concreto del fluido secundario del evaporador) y se aporta calor al fluido secundario del condensador. Las variables manipulables son la velocidad de giro del compresor $N$ y la apertura de la válvula de expansión $A_{v}$, considerándose los caudales de los fluidos secundarios y sus temperaturas de entrada perturbaciones al ciclo. El principal objetivo de control es proporcionar la potencia frigorífica demandada $\dot{Q}_{e}$, lo que puede reflejarse en una referencia para la temperatura de salida del fluido secundario del evaporador $\left(T_{e, s e c, o u t}\right)$. Como se dispone de dos acciones de control, se plantea un segundo objetivo, consistente en producir la potencia frigorífica requerida maximizando la eficiencia energética, descrita mediante el Coeficiente de Comportamiento $(C O P)$, cuya definición se incluye en (1).

$$
C O P=\frac{\dot{Q}_{e}}{\dot{W}_{\text {comp }}}
$$

La eficiencia del evaporador (su capacidad de extraer calor de su fluido secundario), depende principalmente del coeficiente de transferencia de calor. Este es mucho mayor para flujo bifásico que para vapor sobrecalentado, debido al cambio de fase, de forma que se alcanzaría alta eficiencia energética si el flujo de refrigerante en el evaporador fuese totalmente bifásico. Sin embargo, puesto que la salida del evaporador coincide con la aspiración del compresor, se debe evitar la presencia de gotas de refrigerante líquido en la succión del mismo. Esto se consigue tradicionalmente en la industria operando el sistema con un cierto grado de sobrecalentamiento del refrigerante $\left(T_{S H}\right)$ a la salida del evaporador, que se suele mantener bajo para alcanzar un alto $C O P$.

Sin embargo, es posible plantear una optimización global cuya solución sea un ciclo que genere la potencia frigorífica requerida con máxima eficiencia energética. En esta línea, Jain y Alleyne plantean una optimización global que minimiza la exergía destruida [6]; se genera un ciclo óptimo a la vez que se calculan valores óptimos de las variables manipulables, útiles para estrategias de control feedforward. Por su parte, Bejarano et al. proponen un optimizador global no lineal cuyo criterio de eficiencia es el propio $C O P[3,4]$. Sin embargo, la carga computacional de este optimizador no es despreciable, debido a la propia no linealidad del proceso y la exploración exhaustiva necesaria pa- 
Tabla 1: Nomenclatura

\begin{tabular}{|c|c|c|c|}
\hline \multicolumn{2}{|c|}{ Símbolos latinos } & \multicolumn{2}{|c|}{ Símbolos griegos } \\
\hline$A_{v}$ & Apertura de válvula [\%] & $\alpha$ & $\begin{array}{l}\text { Coeficiente de pérdidas por convección } \\
\text { entre el recinto y el ambiente }\left[\mathrm{W} \mathrm{K}^{-1}\right]\end{array}$ \\
\hline$c_{p}$ & $\begin{array}{l}\text { Calor específico a presión constante } \\
{\left[\mathrm{J} \mathrm{kg}^{-1} \mathrm{~K}^{-1}\right]}\end{array}$ & $\rho$ & Densidad $\left[\mathrm{kg} \mathrm{m}^{-3}\right]$ \\
\hline $\begin{array}{l}f \\
G(s)\end{array}$ & $\begin{array}{l}\text { Pendiente de recta de aproximación } \\
\text { Función de transferencia }\end{array}$ & $\omega$ & Frecuencia $\left[\mathrm{rad} \mathrm{s}^{-1}\right]$ \\
\hline$\dot{m}$ & Caudal másico $\left[\mathrm{kg} \mathrm{s}^{-1}\right]$ & \multicolumn{2}{|c|}{ Subíndices y superíndices } \\
\hline$N$ & Velocidad de giro del compresor $[\mathrm{Hz}]$ & $a m b$ & ambiente \\
\hline$P$ & Presión [bar] & $c$ & condensador \\
\hline$\dot{Q}_{e}$ & Potencia frigorífica $[\mathrm{W}]$ & $e$ & evaporador \\
\hline$\dot{Q}_{R}$ & $\begin{array}{l}\text { Potencia térmica de la resistencia } \\
\text { eléctrica }[\mathrm{W}]\end{array}$ & in & entrada \\
\hline$R$ & Resistencia eléctrica & $\min$ & mínimo \\
\hline$T$ & Temperatura $\left[{ }^{\circ} \mathrm{C}\right]$ & nom & nominal \\
\hline$T(s)$ & $\begin{array}{l}\text { Función de sensibilidad } \\
\text { complementaria }\end{array}$ & out & salida \\
\hline$T_{S H}$ & Grado de sobrecalentamiento $\left[{ }^{\circ} \mathrm{C}\right]$ & ref & referencia \\
\hline & Volumen $\left[\mathrm{m}^{3}\right]$ & $s e c$ & fluido secundario \\
\hline$W_{\text {comp }}$ & Potencia mecánica del compresor [W] & tanque & tanque \\
\hline$W_{T}(s)$ & $\begin{array}{l}\text { Ponderación de la función de } \\
\text { sensibilidad complementaria }\end{array}$ & & \\
\hline
\end{tabular}

ra evitar extremos locales. En el presente trabajo se analizan los resultados en régimen permanente del sistema en cuanto a la eficiencia energética y se propone una optimización estática basada en aproximaciones lineales calculadas en base a la característica estática del sistema, de forma que se reduce el tiempo de cálculo en gran medida.

En cuanto al control, la dificultad de controlar este tipo de proceso reside en las altas inercias térmicas, el alto acoplamiento de las variables del sistema y su carácter fuertemente no lineal. En este trabajo se plantean dos enfoques de control: control en potencia y control de temperatura de recinto. En el control en potencia, se le exige al ciclo que satisfaga una cierta demanda de frío $\dot{Q}_{e}^{r e f}$, la cual, dada una cierta temperatura de entrada del fluido secundario del evaporador $T_{e, s e c, \text { in }} \mathrm{y}$ un cierto caudal $\dot{m}_{e, s e c}$, se refleja en una referencia para $T_{e, s e c, o u t}$, de acuerdo a (2).

$$
T_{e, s e c, \text { out }}^{r e f}=T_{e, s e c, \text { in }}-\frac{\dot{Q}_{e}^{r e f}}{c_{p, e, s e c} \dot{m}_{e, s e c}}
$$

Las técnicas lineales de control más utilizadas en la literatura son el control descentralizado [8, 17], el control multivariable por desacoplo [14], control LQG [12, 13], control predictivo [5, 10] y control robusto $H_{\infty}[1,7]$. En este trabajo se presenta un controlador robusto descentralizado diseñado con el método de la parametrización afín por cancelación de dinámica, que se prueba en simulación tanto en seguimiento de referencia de potencia, co- mo en rechazo de perturbaciones al ciclo.

En el caso del control de temperatura de recinto, es necesario tener en cuenta también la dinámica del mismo, en el cual hay un cierto volumen de fluido secundario almacenado, del cual se extrae un cierto caudal que se hace pasar por el evaporador del ciclo y se recircula al propio recinto. Para simular la carga térmica se supone que existe un aporte externo de calor al recinto. En este caso $T_{e, s e c, i n}$ se coincidiría con la temperatura del tanque $T_{\text {tanque }}$, supuesta homogeneización de la temperatura del fluido, y es esta la variable a controlar, actuando la potencia frigorífica generada por el ciclo como acción de control para el controlador de la temperatura del recinto. Se propone en este trabajo una estructura de control en cascada, en la cual un controlador externo genera la referencia de potencia para el ciclo, mientras que un controlador interno se encarga de satisfacer dicha demanda mediante la variación de $N$ y $A_{v}$. Se analizan en simulación el seguimiento de referencia de la temperatura del recinto, así como el rechazo de perturbaciones sobre el aporte externo de calor.

Ambas estrategias de control han sido asimismo aplicadas a una planta experimental de refrigeración situada en el Departamento de Ingeniería de Sistemas y Automática de la Universidad de Sevilla, cuya descripción puede consultarse en la bibliografía asociada [2]. El artículo se organiza de la siguiente manera: en la Sección 2 se dan algunos detalles sobre el simulador del ciclo de refrigeración y se describe el modelo térmico del recinto a 
refrigerar. La Sección 3 está dedicada a la optimización estática de las referencias para el control del ciclo, mientras que en las Secciones 4 y 5 se analizan en simulación y experimentalmente los resultados de la aplicación del controlador de potencia frigorífica y de temperatura de recinto, respectivamente. Finalmente, en la Sección 6 se resumen las principales conclusiones y se proponen algunos trabajos futuros.

\section{SIMULADOR DEL SISTEMA}

La Figura 1 muestra el simulador dinámico desarrollado para el ciclo de refrigeración, que se conecta al simulador del recinto a través del fluido secundario.

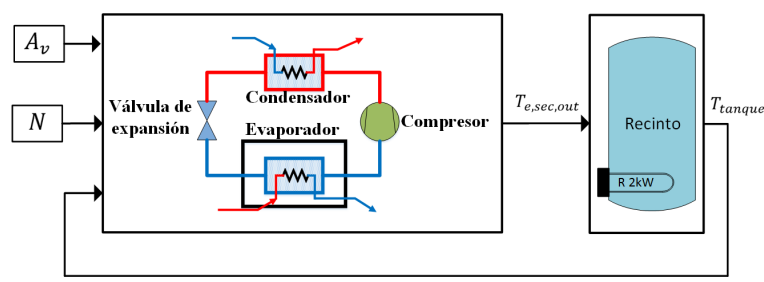

Figura 1: Simulador del ciclo de refrigeración y el recinto a refrigerar

Para el desarrollo de este simulador se ha asumido que la dinámica del condensador es dominante sobre el resto de los componentes del sistema [3]. El modelo térmico del recinto se muestra en (3), donde se aplica un balance de potencia incluyendo la potencia frigorífica generada por el ciclo $\dot{Q}_{e}$, el aporte externo de calor (modelado como una resistencia eléctrica que genera la potencia térmica $\left.\dot{Q}_{R}\right)$ y las pérdidas térmicas con el ambiente.

$$
\begin{aligned}
& \rho_{e, s e c} c_{p, e, s e c} V_{\text {tanque }} \frac{d T_{\text {tanque }}}{d t}= \\
& =\dot{Q}_{R}-\dot{Q}_{e}-\alpha\left(T_{\text {tanque }}-T_{a m b}\right)
\end{aligned}
$$

Las pérdidas térmicas se consideran pequeñas en comparación con las otras potencias, de forma que se desprecian en los cálculos siguientes. El sistema cuenta con dos entradas manipulables que se corresponden con el porcentaje de apertura de la válvula de expansión $A_{v} \in[10,100] \%$ y la velocidad de giro del compresor $N \in[30,50] \mathrm{Hz}$. Como variables de interés se tienen $T_{e, s e c, o u t}, T_{e, s e c, \text { in }}$ (que será considerada como la temperatura del recinto $T_{\text {tanque }}$, ya que en la planta experimental a partir de la que se ha desarrollado el simulador no se tienen medidas directas de la temperatura del recinto, como se muestra en la Figura 2), $T_{S H}$ y $\dot{Q}_{e}$. Como perturbaciones del sistema se tienen $T_{c, s e c, i n}, \dot{m}_{c, s e c}, P_{c, s e c}, \dot{m}_{e, s e c}, P_{e, s e c}, T_{a m b}$ y $\dot{Q}_{R}$, cuyos valores nominales, mínimos y máximos esperados se recogen en la Tabla 2. El simulador del ciclo ha sido ajustado a la planta experimental citada anteriormente mediante identificación en régimen permanente de los parámetros de cada uno de los elementos del ciclo [11].

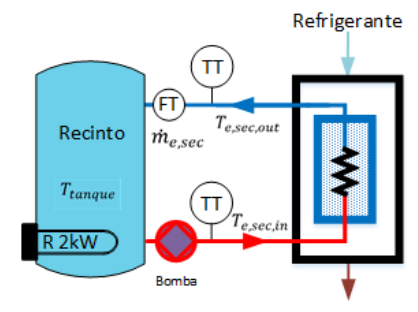

Figura 2: P\&ID del recinto experimental

Tabla 2: Condiciones nominales de las perturbaciones al sistema y valores mínimos y máximos considerados.

\begin{tabular}{cccc}
\hline Perturbación & $\begin{array}{c}\text { Valor } \\
\text { nominal }\end{array}$ & $\begin{array}{c}\text { Valor } \\
\text { mínimo }\end{array}$ & $\begin{array}{c}\text { Valor } \\
\text { máximo }\end{array}$ \\
\hline$T_{c, s e c, i n}\left[{ }^{\circ} \mathrm{C}\right]$ & 30 & 27 & 33 \\
\hline$\dot{m}_{c, s e c}\left[\mathrm{~kg} \mathrm{~s}^{-1}\right]$ & 0.150 & 0.125 & 0.175 \\
\hline$P_{c, s e c}[\mathrm{bar}]$ & 1 & - & - \\
\hline$\dot{m}_{e, s e c}\left[\mathrm{~kg} \mathrm{~s}^{-1}\right]$ & 0.0645 & 0.0545 & 0.0745 \\
\hline$P_{e, s e c}[\mathrm{bar}]$ & 1 & - & - \\
\hline$T_{a m b}\left[{ }^{\circ} \mathrm{C}\right]$ & 25 & - & - \\
\hline$\dot{Q}_{R}[\mathrm{~W}]$ & - & 0 & 2000 \\
\hline
\end{tabular}

\section{OPTIMIZACIÓN ESTÁTICA}

Se han realizado simulaciones bajo distintas condiciones de perturbaciones y de variables manipulables del ciclo, considerado los rangos indicados en la Tabla 2. En la Figura 3 se muestran los valores de $C O P$ en equilibrio para una cierta apertura de válvula constante e imponiendo diferentes valores de las perturbaciones al ciclo. Esta gráfica debe analizarse cualitativamente, ya que la tendencia es idéntica para todo el rango de $A_{v}$. Se observa que, para las condiciones de funcionamiento evaluadas, el valor máximo de $C O P$ se alcanza para la velocidad mínima de compresor.

Por otro lado, en la Figura 4 se muestra la variación de la potencia frigorífica para valores nominales de las perturbaciones e imponiendo diferentes valores de las variables manipulables, donde se pueden apreciar el rango de potencia que se consigue para cada velocidad del compresor. Se observa que, dada una cierta potencia frigorífica, esta puede generarse con todo el rango del compresor, excepto en los extremos del intervalo admisible de potencias. De este modo, la forma óptima de satisfacer una cierta demanda de frío es imponer la mínima velocidad del compresor siempre que sea posible. Esta imposición se realiza de cara al control mediante la referencia de $T_{S H}$, ya que la re- 


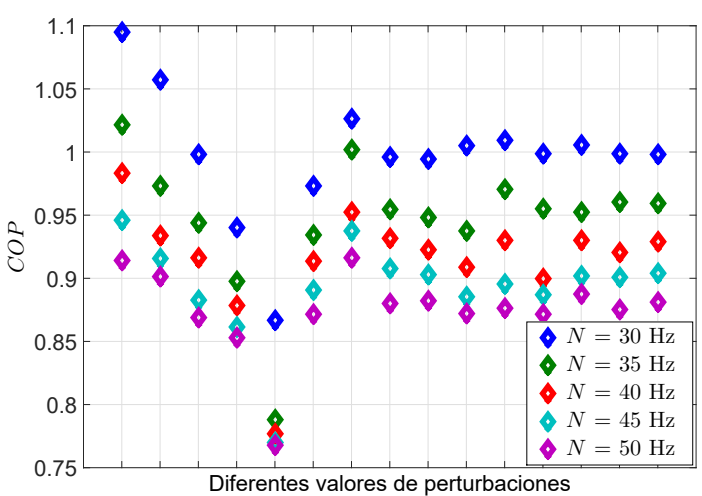

Figura 3: $C O P$ en régimen permanente para diferentes valores de perturbaciones, con $A_{v}$ constante

ferencia de $\dot{Q}_{e}$ o equivalentemente $T_{e, \text { sec,out }}$ viene impuesta por la demanda de frío.

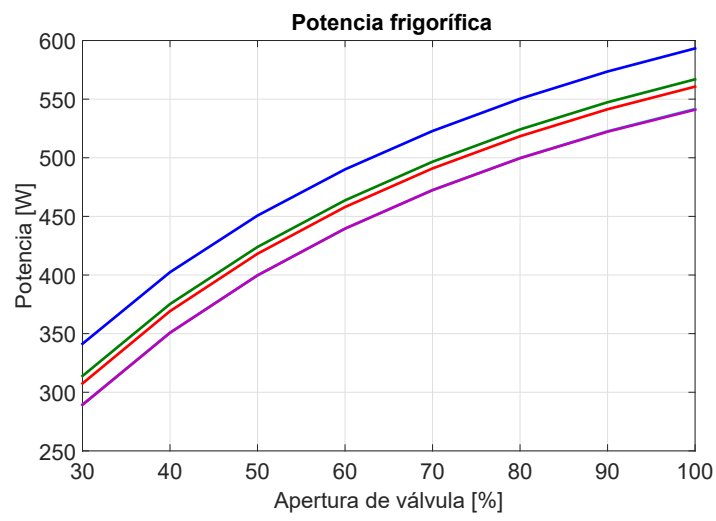

Figura 4: Rango de potencia frigorífica generada

En base a las conclusiones extraídas se ha diseñado un generador de referencia para el grado de sobrecalentamiento que fuerza al ciclo a una velocidad de compresor mínima para la obtención de la potencia frigorífica deseada. Para estimar la consigna $T_{S H}^{r e f}$ se ha realizado el siguiente método en base a datos de simulación. Manteniendo constante $N=30 \mathrm{~Hz}$, se ha barrido todo el rango admisible de $A_{v}$ con diferentes valores de las perturbaciones $T_{c, s e c, i n}, \dot{m}_{c, s e c}, T_{e, s e c, i n} \mathrm{y} \dot{m}_{e, s e c}$, despreciando esta última al no producir variaciones apreciables sobre $T_{S H}$. Una vez recogidos los valores de $T_{S H}$ para los rangos indicados en la Tabla 2 , se han buscando relaciones, mediante rectas con pendiente variable que han sido a su vez calculadas mediante simulación, con cada una de las perturbaciones y, en base a un punto de equilibrio precalculado para las condiciones nominales del sistema, se estima $T_{S H}^{r e f}$ como la suma de un valor nominal conocido y la aportación de cada una de las variaciones de las perturbaciones con respecto a las condiciones nominales, como se indica en (4). En la Figura 5 se muestran algunos resultados generados por el estimador en comparación con los que genera el optimizador no lineal citado en la Sección 1 como referencias óptimas [4].

$$
\begin{aligned}
T_{S H}^{r e f} & =T_{S H, \text { nom }}+ \\
& +f\left(\dot{m}_{c, s e c}\right)\left(\dot{m}_{c, s e c}-\dot{m}_{c, s e c, \text { nom }}\right) \\
& +f\left(T_{c, \text { sec }, \text { in }}\right)\left(T_{c, s e c, \text { in }}-T_{c, \text { sec }, \text { in }, \text { nom }}\right) \\
& +f\left(T_{e, \text { sec }, \text { in }}\right)\left(T_{e, \text { sec }, \text { in }}-T_{e, s e c, \text { in }, \text { nom }}\right)
\end{aligned}
$$

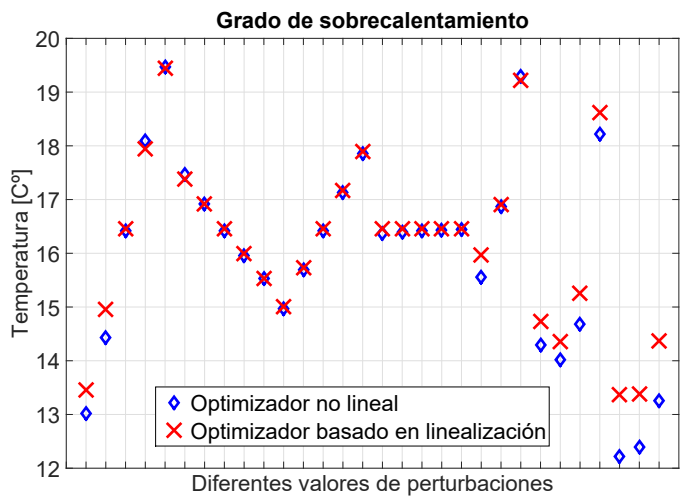

Figura 5: Estimador de valores admisibles de $T_{S H}$ para $N=30 \mathrm{~Hz}$

\section{CONTROL EN POTENCIA}

\subsection{RESULTADOS DE SIMULACIÓN}

Se utilizan técnicas de control robusto para diseñar un controlador descentralizado, donde $T_{e, \text { sec,out }}$ se controla manipulando $A_{v}, \mathrm{y} T_{S H}$ se controla mediante $N$. En base a una función de transferencia nominal $G_{n o m}(s)$, se consideran incertidumbres estructurales debido a la dinámica de evaporador, la cual, como se comentó en la Sección 2, había sido despreciada. Se han considerado también incertidumbres paramétricas en $G_{n o m}(s)$ debido a cómo se ve afectado el sistema según el punto de equilibrio del que parte. Posteriormente se ha definido la ponderación $W_{T_{T_{e, s e c} \text { out }}}(s)$ de la función de sensibilidad complementaria $T_{T_{e, s e c, \text { out }}}(s)$ para $T_{e, s e c, \text { out }}$ con respecto a $A_{v}$, así como la ponderación $W_{T_{T_{S H}}}(s)$ de la función de sensibilidad complementaria $T_{T_{S H}}(s)$ de $T_{S H}$ con respecto a $N$, de forma que se cumplan los criterios de robustez, como se muestra en las Figuras 6 y 7. Aplicando parametrización afín con cancelación de dinámica se han obtenidos los controladores desacoplados correspondientes.

Además, ya que el objetivo del optimizador, para una cierta demanda de frío, es calcular el valor de $T_{S H}^{r e f}$ tal que la velocidad del compresor sea mínima en régimen permanente, se ha realizado 


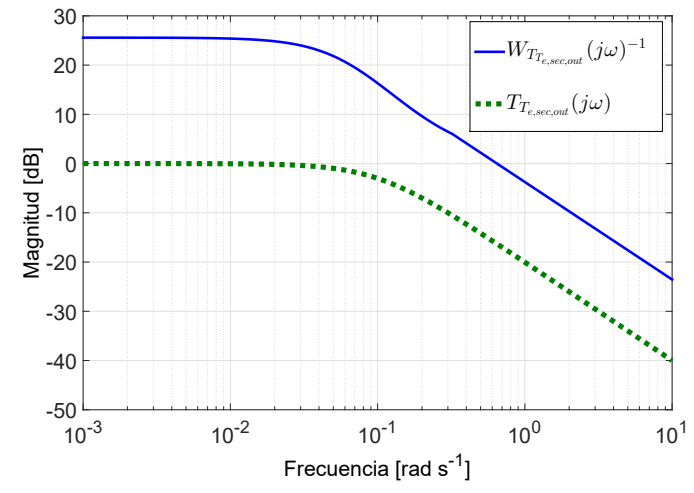

Figura 6: Ganancia de la función de sensibilidad complementaria $T_{T_{e, \text { sec out }}}(s)$ de $T_{e, \text { sec,out }}$ respecto a $A_{v}$ y su ponderación $W_{T_{T_{e}, s e}}$ $(s)$

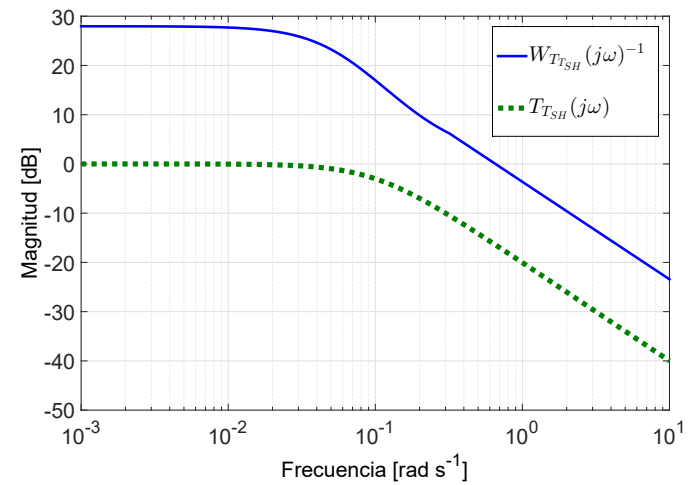

Figura 7: Ganancia de la función de sensibilidad complementaria $T_{T_{S H}}(s)$ de $T_{S H}$ respecto a $N$ y su ponderación $W_{T_{T_{S H}}}(s)$

una comparativa entre controlar $T_{e, s e c, o u t ~}$ y $T_{S H}$ simultáneamente, y controlar solo $T_{e, s e c, o u t}$ dejando libre $T_{S H}$ y fijando el compresor a su velocidad mínima. Para ello se han simulado ambos casos bajo el mismo perfil de perturbaciones mostrado en la Figura 8 y aplicando los cambios de referencia de $Q_{e}$ mostrados en la Figura 9.

La Figura 10 muestra la evolución de $T_{e, s e c, o u t}$ y $T_{S H}$, cuando se controla explícitamente $T_{S H}$ y cuando se deja libre, mientras que la Figura 11 muestra el $C O P$ alcanzado en ambos casos. Se puede observar que cuando se alcanza el equilibrio, el resultado entre controlar $T_{S H}$ y dejarlo libre, fijando la velocidad del compresor a su valor mínimo, es prácticamente idéntico. Sin embargo, cuando el sistema experimenta una perturbación el $C O P$ obtenido para el caso del $T_{S H}$ controlado es inferior al caso en que se deja libre, por lo que se llega a la conclusión que para las condiciones de funcionamiento del sistema la forma óptima de satisfacer la demanda de frío es manteniendo fija la velocidad del compresor a su valor mínimo.
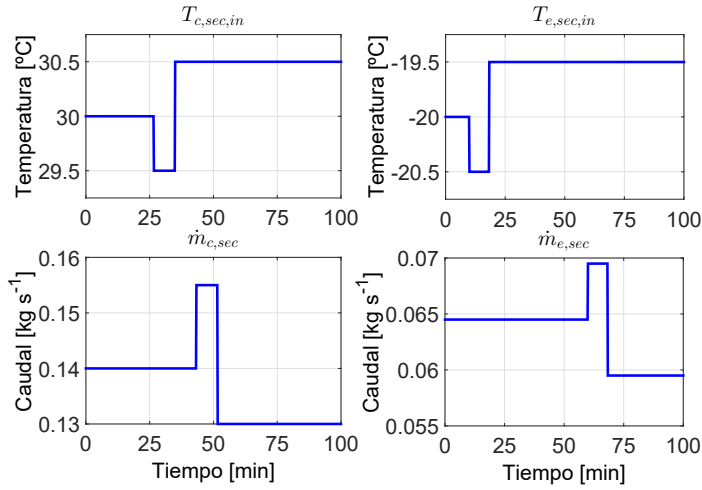

Figura 8: Perfil de perturbaciones

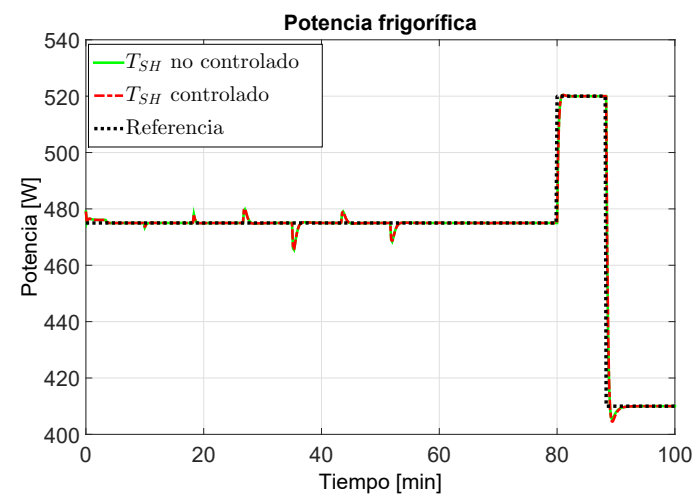

Figura 9: Potencia frigorífica generada

\subsection{RESULTADOS EXPERIMENTALES}

En este apartado se pretenden replicar los resultados de simulación en un experimento real, para lo que se utiliza la planta experimental citada en la Sección 1 [2]. La planta puede configurarse con hasta 2 compresores y hasta 2 evaporadores. Cada evaporador está conectado por un circuito de fluido secundario a un tanque, de forma que se puede trabajar a temperaturas de referencia de $5^{\circ} \mathrm{C}$ y $-20^{\circ} \mathrm{C}$. Se dispone de sensores de temperatura y presión en los puntos característicos del sistema. Además, existe un sensor de caudal para cada fluido secundario, así como una bomba que permite su recirculación. Para simular la carga térmica en los tanques se dispone de dos resistencias eléctricas. El refrigerante utilizado es $R 404 a$, mientras que los fluidos de los circuitos secundarios son una solución acuosa de glicol. El condensador empleado es de flujo cruzado, cuyo fluido secundario es el aire exterior que se mueve mediante un ventilador.

Por motivos técnicos, los experimentos se han realizado en el tanque a $5^{\circ} \mathrm{C}$. Esto implica un punto de funcionamiento diferente al estudiado en la simulación mostrada anteriormente, por tanto el controlador diseñado ha sido reajustado. Además, 

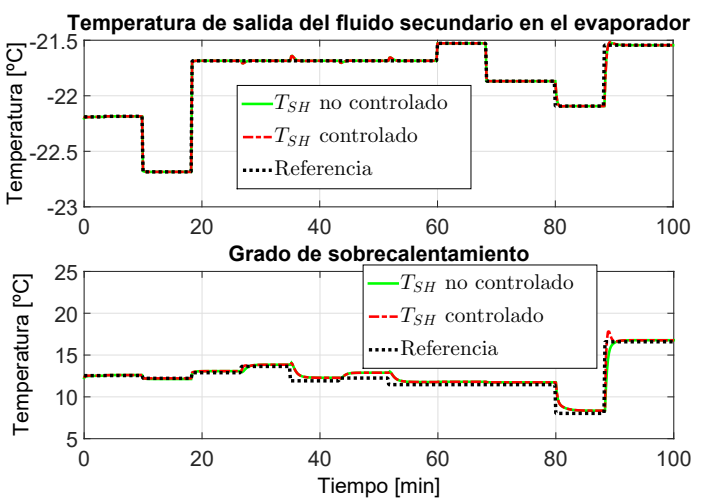

Figura 10: Resultados del control de $T_{e, s e c, o u t} \mathrm{y}$ $T_{S H}$

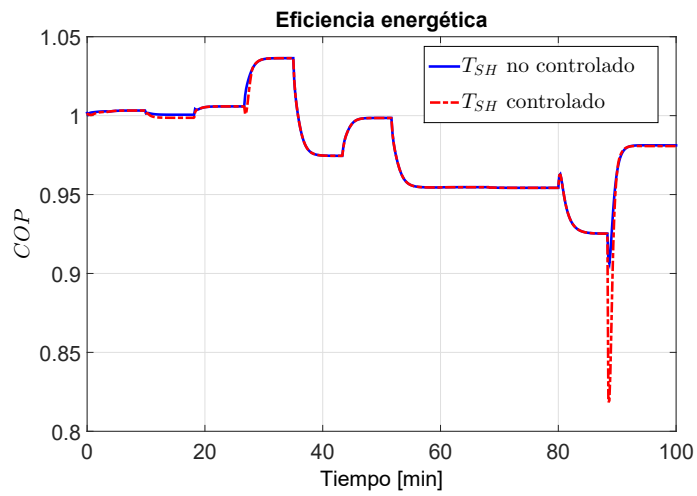

Figura 11: Eficiencia energética

debido al sobredimensionamiento del condensador se ha implementado un controlador todo-nada sobre el ventilador con el fin de ajustar la presión del condensador a la presión obtenida en simulación.

Siguiendo las conclusiones del estudio realizado en simulación, se decide no controlar $T_{S H}$, imponiendo $N=30 \mathrm{~Hz}$. En el experimento de la Figura 12 se replican los dos cambios de referencia de potencia de la Figura 9: el primero en el minuto 51 aproximadamente con un escalón de $50 \mathrm{~W}$, mientras que el segundo se realiza en el minuto 64 con un escalón negativo de 100 W. Para mantener constante a $5^{\circ} \mathrm{C}$ la temperatura de entrada del fluido secundario al evaporador se ha diseñado un controlador que ajusta la potencia térmica aportada por la resistencia eléctrica $\dot{Q}_{R}$.

El rizado que se observa en la potencia frigorífica generada es debido al control todo-nada implementado para el ventilador del condensador, ya que la variación de la presión del condensador afecta a los caudales del ciclo y por tanto a la transferencia de calor del evaporador. En la segunda subfigura se puede observar que el controlador alcanza las referencias pedidas con un tiempo de subida de unos 74 segundos con una sobreoscila-
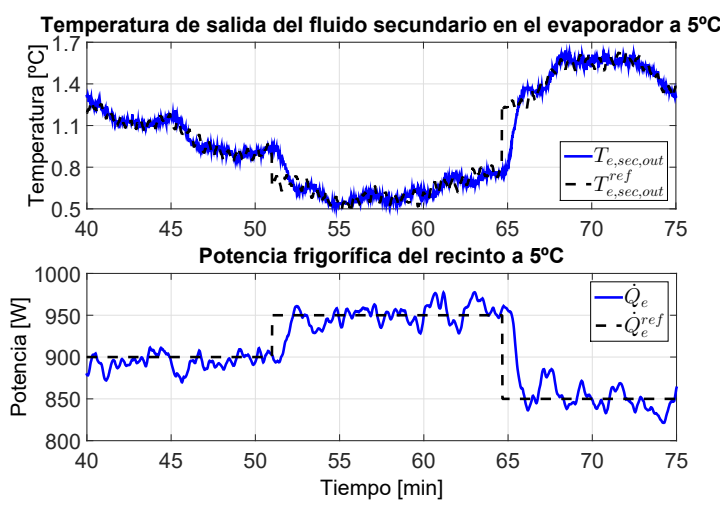

Figura 12: Experimento de control en potencia

ción del $20 \%$. Los resultados de control se pueden considerar suficientes como primera aproximación para el control de este proceso. Asimismo, en la primera subfigura se puede observar que los cambios de referencia de potencia frigorífica provocan cambios de referencia sobre $T_{e, s e c, o u t}$, que es la variable realmente controlada mediante $A_{v}$.

\section{CONTROL DE TEMPERATURA DE RECINTO}

\subsection{RESULTADOS DE SIMULACIÓN}

Una vez diseñado el control de potencia se aplica la generación de frío para controlar la temperatura de un recinto. Para ello, como muestra la Figura 1, se ha añadido el modelo térmico del recinto basado en (3). Se propone la estrategia de control en cascada mostrada en la Figura 13. Dada $T_{\text {tanque }}^{\text {ref }}$ y conocida $T_{\text {tanque }}$, la cual es indirectamente medida, se calcula el valor de potencia deseada $\dot{Q}_{e}^{r e f}$ mediante el controlador externo PI. Una vez conocida la referencia de potencia, el controlador interno se corresponde con el desarrollado en la Sección 4, el cual en base a las conclusiones extraídas se realizará únicamente controlando $T_{e, s e c, o u t}$ por medio de $A_{v}$ y manteniendo el compresor a su velocidad mínima y dejando $T_{S H}$ libre. En la Figura 14 se pueden observar los resultados del control de la temperatura del recinto y la potencia como acción de control del controlador externo.

\subsection{RESULTADOS EXPERIMENTALES}

Se replica experimentalmente el control en cascada diseñado y aplicado en los resultados de simulación obtenidos en la Figura 14. De manera análoga al primer experimento, se han adaptado las ganancias del controlador para el recinto a $5^{\circ} \mathrm{C}$ de la planta experimental y se ha utilizado un con- 


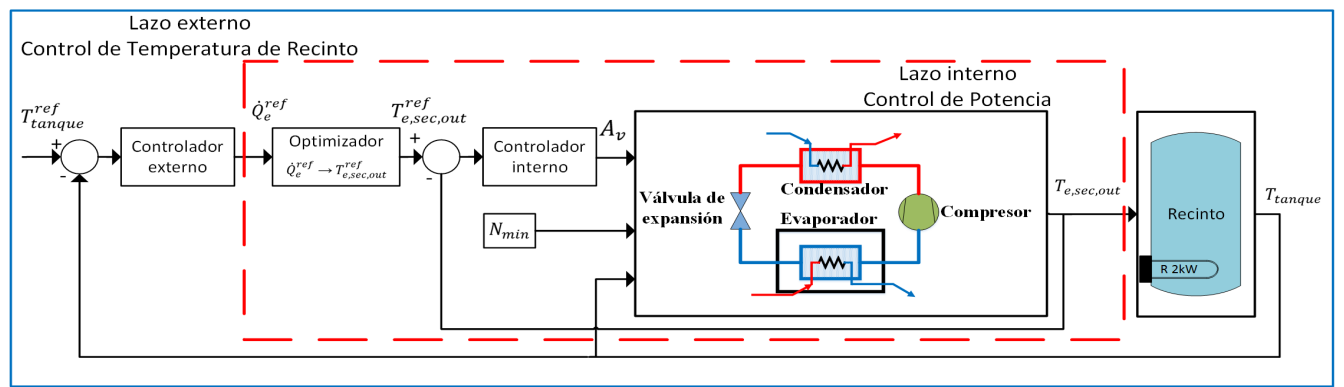

Figura 13: Estructura de control en cascada para la temperatura del recinto a refrigerar
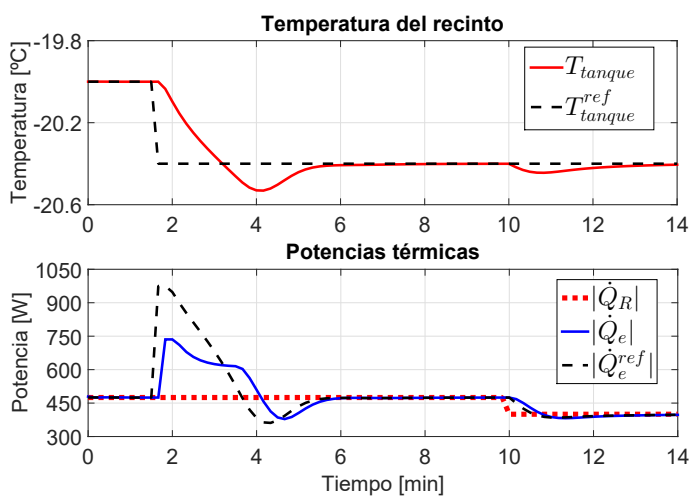

Figura 14: Resultados de simulación del control en cascada de la temperatura del recinto

trol todo-nada para la presión del condensador. En este caso la carga térmica del recinto simulada mediante la resistencia eléctrica actúa como una perturbación a la temperatura del recinto.

En la Figura 15 se muestran los resultados experimentales obtenidos, donde se realiza un cambio de referencia en la temperatura del tanque en el minuto 57 aproximadamente de $-0.4^{\circ} \mathrm{C}$, mientras que se impone una reducción del $10 \%$ en la potencia térmica aportada por la resistencia en el minuto 87 aproximadamente.
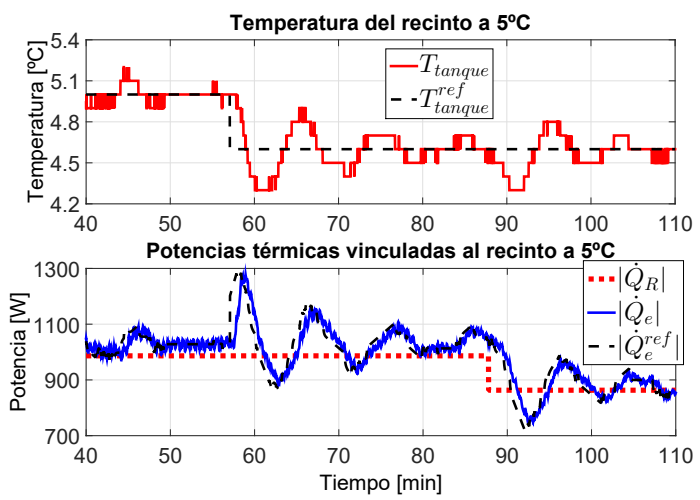

Figura 15: Resultados experimentales del control en cascada de la temperatura del recinto
En la primera subfigura se muestra la temperatura del tanque y su referencia, mientras que en la segunda aparecen la potencia frigorífica generada por el ciclo y su referencia (consecuencia del control en cascada), así como la potencia térmica generada por la resistencia. En el cambio de referencia se obtiene un tiempo de subida de 130 segundos y una sobreoscilación del $70 \%$, mientras que se puede observar cómo el controlador rechaza la perturbación en la temperatura y cómo la potencia frigorífica se adapta rápidamente al cambio aplicado sobre la resistencia. Estos resultados preliminares significan un punto de partida para mejorar el control en futuros experimentos.

\section{CONCLUSIONES Y TRABAJOS FUTUROS}

En este artículo se ha presentado un optimizador estático para la referencia del grado de sobrecalentamiento de un ciclo de refrigeración. En base al análisis estático del comportamiento del ciclo, se han generado una serie de aproximaciones lineales que permiten calcular el sobrecalentamiento correspondiente a la máxima eficiencia del sistema para una cierta demanda de frío, que coincide en un rango amplio de condiciones de la planta con la velocidad mínima del compresor.

Respecto al control de potencia, se ha comparado el desempeño en dos situaciones de un controlador robusto descentralizado diseñado con el método de la parametrización afín por cancelación de dinámica: por un lado controlando la potencia a través de la temperatura de salida del fluido secundario del evaporador y el grado de sobrecalentamiento, y por otro lado controlando solo la potencia y fijando la velocidad del compresor a su valor mínimo. Se concluye que, sobre todo en el rechazo de perturbaciones, la eficiencia alcanzada es muy similar en ambos casos, incluso algo mejor en el controlador que deja libre el sobrecalentamiento. Se ha aplicado este controlador experimentalmente y se obtienen resultados preliminares que pueden ser mejorados mediante el reajuste de ganancias. Con 
vista a un sistema real se propone en cualquier caso la implementación de un control de supervisión del grado de sobrecalentamiento, de forma que solo actúe cuando este alcanza valores cercanos a $0^{\circ} \mathrm{C}$, y cuya función sería únicamente llevar el sobrecalentamiento a una zona segura de trabajo, sacrificando el objetivo de maximizar la eficiencia.

Respecto al control de temperatura, se propone una estrategia de control en cascada en la cual la potencia resulta ser la acción de control de un controlador jerárquicamente superior que regula la temperatura del recinto. Esta estrategia ha sido aplicada también experimentalmente, obteniendo resultados preliminares que igualmente pueden ser mejorados mediante reajuste de los controladores.

Como trabajo futuro, se propone reajustar los controladores experimentales así como extender el estudio de eficiencia a sistemas con múltiples recintos y/o múltiples compresores, pudiendo ser de interés el control de la velocidad del compresor.

\section{Agradecimientos}

Los autores quieren expresar su agradecimiento al Ministerio de Ciencia e Innovación por la financiación de este trabajo, a través de los proyectos DPI2015-70973-R y DPI2016-79444-R.

\section{Referencias}

[1] J. A. Alfaya, G. Bejarano, M. G. Ortega, and F. R. Rubio. Controllability analysis and robust control of a one-stage refrigeration system. Eur. J. of Control, 26:53-62, 2015.

[2] G. Bejarano, J. A. Alfaya, M. G. Ortega, and F. R. Rubio. Design, automation and control of a two-stage, two-load-demand experimental refrigeration plant. In $23^{\text {rd }}$ Mediterranean Conf. on Control and Autom., Torremolinos (Spain), pages 537-544, 2015.

[3] G. Bejarano, J. A. Alfaya, M. G. Ortega, and M. Vargas. On the difficulty of globally optimally controlling refrigeration systems. Appl. Therm. Eng., 111:1143-1157, 2017.

[4] G. Bejarano, M. G. Ortega, and F. R. Rubio. Optimización global estática de sistemas de refrigeración. In XXXVII Jorn. de Autom., Madrid (Spain), pages 19-26, 2016.

[5] H. Fallahsohi, C. Changenet, S. Placé, C. Ligeret, and X. Lin-Shi. Predictive functional control of an expansion valve for minimizing the superheat of an evaporator. Int. J. of Refrig., 33(2):409-418, 2010.

[6] N. Jain and A. G. Alleyne. A framework for the optimization of integrated energy systems. Appl. Therm. Eng., 48:495-505, 2012.
[7] L. S. Larsen and J. R. Holm. Modelling and multi-variable control of refrigeration systems. ECOS 2003, 2003.

[8] J. Marcinichen, T. del Holanda, and C. Melo. A dual SISO controller for a vapor compression refrigeration system. In Int. Refrig. and Air Cond. Conf., pages 2444, 1-8, 2008.

[9] L. Pérez-Lombard, J. Ortiz, and C. Pout. A review on buildings energy consumption information. Energy and Build., 40(3):394-398, 2008.

[10] N. L. Ricker. Predictive hybrid control of the supermarket refrigeration benchmark process. Control Eng. Pract., 18(6):608-617, 2010.

[11] D. Rodríguez, G. Bejarano, J. A. Alfaya, M. G. Ortega, and F. Castaño. Parameter identification of a multi-stage, multi-loaddemand experimental refrigeration plant. Control Eng. Pract., 60:133-147, 2017.

[12] L. C. Schurt, C. J. L. Hermes, and A. TrofinoNeto. A model-driven multivariable controller for vapor compression refrigeration systems. Int. J. of Refrig., 32(7):1672-1682, 2009.

[13] L. C. Schurt, C. J. L. Hermes, and A. TrofinoNeto. Assessment of the controlling envelope of a model-based multivariable controller for vapor compression refrigeration systems. Appl. Therm. Eng., 30(13):1538-1546, 2010.

[14] Y. Shen, W.-J. Cai, and S. Li. Normalized decoupling control for high-dimensional MIMO processes for application in room temperature control HVAC systems. Control Eng. Pract., 18(6):652-664, 2010.

[15] US Energy Information Administration. Residential energy consumption survey (RECS). Technical report, Energy Inf. Adm., Washington D.C, USA, 2009.

[16] US Environmental Protection Agency. National action plan for energy efficiency: Sector collaborative on energy efficiency accomplishments and next steps.

[17] J. Wang, C. Zhang, Y. Jing, and D. An. Study of neural network PID control in variable-frequency air-conditioning system. In IEEE Int. Conf. on Control and Autom., pages 317-322, 2007. 